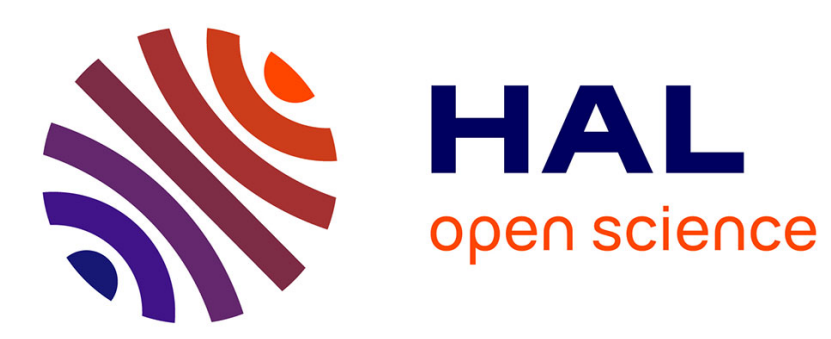

\title{
Les sens des circulations: les sens vécu, objectivé et pratique des mobilités spatiales
}

Benoît Feildel, Denis Martouzet

\section{To cite this version:}

Benoît Feildel, Denis Martouzet. Les sens des circulations: les sens vécu, objectivé et pratique des mobilités spatiales. RTS - Recherche Transports Sécurité, 2014, Les sens des circulations, 2014 (02-03), pp.121-124. 10.4074/S0761898014002040 . halshs-01084376

\section{HAL Id: halshs-01084376 \\ https://shs.hal.science/halshs-01084376}

Submitted on 14 Mar 2018

HAL is a multi-disciplinary open access archive for the deposit and dissemination of scientific research documents, whether they are published or not. The documents may come from teaching and research institutions in France or abroad, or from public or private research centers.
L'archive ouverte pluridisciplinaire HAL, est destinée au dépôt et à la diffusion de documents scientifiques de niveau recherche, publiés ou non, émanant des établissements d'enseignement et de recherche français ou étrangers, des laboratoires publics ou privés. 


\section{Les sens des circulations : les sens vécu, objectivé et pratique des mobilités spatiales}

\section{The sense of movements: lived, objectified and practical meanings of spatial mobilities}

\section{Benoît Feildel · Denis Martouzet}

(c) IFSTTAR et Éditions NecPlus 2014

Quel sens confère-t-on à la mobilité spatiale ? L'analyse des déplacements par les chercheurs correspond-elle à la signification qu'en donnent ceux qui se déplacent ? Comment prendre en compte les sens des circulations dans l'organisation des territoires ? Si la question des sens du mouvement n'est pas nouvelle en soi, et si certains ouvrages ayant fait date [1] ont contribué à souligner l'acuité d'une telle interrogation dans nos sociétés de la modernité avancée, le présent dossier de la revue Recherche Transports Sécurité cherche avant tout à croiser les différentes approches d'un même objet. Comme à travers un kaléidoscope, il cherche à faire voir les différents sens des mobilités. En confrontant le sens vécu, tel qu'il est construit par les individus en mouvement, le sens objectivé, rapporté par l'observateur extérieur cherchant à comprendre, et le sens pratique, celui qui guide l'action publique pour organiser ces mêmes circulations, il s'agit de mesurer plus précisément les écarts entre ces différents sens, pour en comprendre les raisons, mais aussi mieux en apprécier les conséquences. Le simple fait de la juxtaposition de ces différents points de vue a déjà ici valeur heuristique. Ce que nous révèlent les différentes contributions à ce dossier thématique est la polysémie des sens attachés à la mobilité et la nécessité, dès lors, d'interroger de façon systématique les différents rapports : celui du chercheur à son objet, celui du politique à ses institutions et à ses administrés, mais aussi et surtout, en premier ressort, celui de l'habitant à sa propre mobilité.

«Quel sens confère-t-on à la mobilité spatiale? » Cette interrogation, posée dans l'appel à communications pour l'atelier intitulé « Les sens des circulations. Les sens vécu,

Benoît Feildel $(\bowtie)$

École Polytechnique de l'Université de Tours, UMR 7324 CITERES, 35 allée Ferdinand de Lesseps, 37200 Tours

e-mail : benoit.feildel@univ-tours.fr

Denis Martouzet $(\triangle)$

École Polytechnique de l'Université de Tours, UMR 7324 CITERES, 35 allée Ferdinand de Lesseps, 37200 Tours

e-mail : denis.martouzet@univ-tours.fr objectivé et pratique des mobilités spatiales », dont émanent les textes du présent dossier, et qui était organisé dans le cadre du $11^{\mathrm{e}}$ congrès de la Société Internationale d'Ethnologie et de Folklore [2], mérite qu'on s'y arrête pour différentes raisons : pour préciser cette interrogation tout d'abord, mais surtout pour restituer toute l'extension qu'elle a pu prendre lors des débats nourris pendant cet atelier. Pour cela, quatre des mots qui composent cette interrogation sont à questionner : « sens », « on », « mobilité » et l'adjectif « spatiale» qui qualifie cette dernière.

Tout d'abord, sans entrer trop avant dans les explications relevant de la linguistique, le terme de « sens » soulève la question de son propre sens. Le jeu sur la polysémie du terme «sens », pouvant signifier à la fois la finalité, l'orientation, ou encore la maîtrise des compétences (« avoir le sens de...»), dénote bien les ambiguïtés qu'il convient ainsi de soulever, pour que la précision l'emporte sur la confusion. Le terme de «sens » s'oppose, en le complétant, à celui de signification [3]. Celle-ci ressortit plutôt de la définition, nécessairement générale et de ce fait décontextualisée de toute circonstance qui amènerait à la nuancer. Partant, la signification acquiert une valeur d'objectivité, à ceci près que l'énonciateur de cette définition n'est pas nécessairement parfaitement objectif quant aux choix auxquels il procède dans la construction de la définition du terme à définir. La signification d'un terme est ce qu'il donne à penser en tant que signe. Le sens, lui, est contextualisé : l'énonciation d'un terme ou d'une expression prend tout son sens par le contexte dans lequel il s'inscrit et ce contexte est bien, étymologiquement parlant, dans le texte lui-même qui insère et contient le terme, lui conférant ainsi une certaine épaisseur, une teinte particulière. Mais ce contexte est aussi d'ordre psychologique, relationnel, social et culturel : le locuteur inscrit sa parole dans le rapport qu'il a avec lui-même, dans le rapport qu'il a avec le récepteur, dans le rapport qu'il a avec le tout qui l'englobe, et ces rapports sont tout autant composés d'éléments tangibles et observables que d'éléments représentationnels. C'est donc bien ici du sens des circulations et non de leur signification dont il s'agit, même si cette dernière intervient nécessairement dans la 
construction du sens. Si l'on considère ainsi que le sens est plus large que la signification, que le sens est dynamique et contextualisé par opposition au caractère statique de la signification, que le sens est donc de l'ordre du parcours dans la mesure où on peut renvoyer à une toujours possible intention, alors pour être tout à fait juste il aurait fallu écrire au pluriel : quels sens confère-t-on à la mobilité spatiale ?

Ce pluriel se cache en fait implicitement derrière le « on » qui a été utilisé à dessein. Ce « on » est d'abord la personne elle-même qui se déplace : il s'agit alors de placer celle-ci dans une posture réflexive pour saisir le sens que la personne donne à sa propre mobilité. Qu'est-ce qui la conduit à se déplacer ? Et de cette manière ? Seule ou avec tel autre ? Usant d'un mode technique de déplacement ? À tel moment de la journée ou de l'année ? Qu'est-ce qui, en lui-même (ses compétences et la représentation qu'il s'en fait) et en contexte (que lui proposent le territoire, la société et leurs composantes), lui permet de circuler ? Les questions sont multiples. Les routines permettent d'éviter d'avoir à y répondre systématiquement. L'objectif du chercheur est à la fois de comprendre ces routines, de les analyser [4], mais aussi de les dépasser car tout déplacement n'est pas que simple routine [5]. Le chercheur est donc le deuxième contenu implicite de ce «on ». Un troisième serait les pouvoirs publics dans leur dimension analytique et décisionnelle et/ou leurs représentants, tout aussi analytiques mais plus techniques, mettant en œuvre des politiques décidées en amont, fabriquant le territoire et accompagnant ceux qui se déplacent. Quelles représentations les chercheurs, les pouvoir publics et leurs représentants se font des habitants et usagers des territoires. Autrement dit : quels sens confèrent-ils à leur mobilité ?

On l'a compris, la mobilité dont il est question ici n'est aucunement réductible aux déplacements. Ceux-ci la construisent et, en retour, la mobilité, par l'épaisseur, le sens qu'elle donne aux déplacements influe ceux-là. Un rapport s'établit ainsi entre déplacements et mobilité, les premiers constituant la dimension objective de la deuxième. Les déplacements sont mesurables, qualifiables, objectifs ou objectivables. La mobilité relève nécessairement de l'interprétation qui, aussi étayée soit-elle, laisse une large marge à différentes interprétations plus ou moins contradictoires, plus ou moins compatibles et complémentaires. Ainsi, l'analyse comportementale des individus, leurs choix, leurs pratiques et actions mais aussi leurs dires nous renseignent sur le sens qu'ils donnent à leurs déplacements et au système que ceux-ci forment. Mais, dans un second temps, il faut bien considérer que ce sens ainsi saisi donne des informations sur des éléments relatifs à la personne et ressortissant de la compréhension qu'il a des territoires et de leur fonctionnement, de la société, d'autrui et d'elle-même. La mobilité, comme sens des déplacements, donne sens à l'ensemble de ces différents rapports au territoire, à la société, à l'altérité, à soi. La mobilité est révélatrice des représentations que les personnes s'en font.

L'adjectif «spatiale » appliqué à la mobilité, toujours dans cette question adressée aux auteur(e)s, permet de cibler mais ne doit pas enfermer. Il s'agit bien du sens donné aux déplacements de personnes, excluant ainsi la mobilité sociale, du moins comme approche première puisque nombreux sont les travaux mettant en évidence le lien entre ces deux types de mobilité [6]. Le recours à la métaphore de la circulation dans l'intitulé plus général du dossier, empruntée à la mécanique des fluides, n'est pas simplement rhétorique mais à entendre en un sens méthodologique. À l'origine, la circulation qualifie le mouvement d'un fluide en circuit fermé puis, par analogie, un déplacement de personnes, de véhicules sur des voies - de circulation justement. Le terme de « circulation » permet de circonscrire plus précisément le champ de la mobilité, en le restreignant au domaine de la quotidienneté et du mouvement observable, des déplacements et de leur déroulement. Cela étant, même s'il est principalement question de mouvements quotidiens dans l'espace, dans différents espaces (urbains, périurbains et ruraux), dans différents contextes (familial, professionnel, institutionnel), l'ensemble des dimensions de la mobilité (représentationnelle, technique, sociale, résidentielle) ne peut être exclu de la compréhension et de l'analyse du sens des déplacements, dans la mesure où l'ensemble de ces dimensions participe à la construction de ce sens. De même, si la mobilité est d'abord regardée par une entrée spatiale, cela ne signifie aucunement que ce sens pourrait être strictement qualifié de spatial. Au contraire, il renvoie aussi au rapport au temps, au rapport aux pouvoirs, à l'autorité, aux valeurs, etc., à la société dans ses différentes dimensions. La mobilité renvoie aussi à soi-même : l'analyse du sens a aussi valeur de miroir dans la mesure où parler de la mobilité que l'on déploie ou que l'on construit revient aussi, en même temps, à parler de soi. Enfin, s'il est avant tout question de mobilité quotidienne dans ce recueil de textes, celle-ci se trouve nécessairement enchâssée dans une mobilité à une échelle plus large, intégrant les trajectoires résidentielle et sociale, et débordant ainsi la stricte logique circulatoire.

Questionner la mobilité c'est aussi interroger l'immobilité dont le sens est, quant à lui, en partie contenu dans le terme d'ancrage. Par la mise en perspective de ces deux termes, mobilité et ancrage [7, 8, 9, 10], c'est plus largement du rapport, plus ou moins labile, de l'humain à ses espaces de vie dont il est question, autrement dit son mode d'habiter [11, 12]. Loin d'être opposés, les deux termes sont liés dans un rapport dialectique. Ainsi, vouloir appréhender le sens des mobilités implique nécessairement d'interroger les ancrages des individus, les multiples formes, temporelles et spatiales, statiques mais aussi 
dynamiques, de leur fixation en un certain nombre de lieux, de leur appartenance à un certain nombre de territoires. De même, réciproquement, l'ancrage, comme expression de l'immobilisme, ne saurait être appréhendé sans considérer le fait qu'il est à la fois la condition de réalisation et le point d'achèvement des mobilités [10]. Alors que la mobilité est devenue une norme - et l'immobilité, dans certains cas, une revendication - il s'agit d'interroger, sans céder aux facilités de l'indistinction, les interactions entre le statique et le mouvant, et les sens qu'elles prennent.

Ce dossier thématique s'organise dès lors en trois parties, cherchant chacune à éclairer l'un de ces trois sens de la mobilité, sens vécu, objectivé ou pratique, en intégrant pour cela le contrepoint de l'un, de l'autre, ou des deux à la fois.

La première partie, à travers deux contributions, s'attache ainsi à la question du sens des circulations, construit par les individus en mouvement, préférentiellement dans des espaces réputés problématiques du point de vue des mobilités : les espaces périurbains. Le premier texte, de Benjamin Pradel et al., interroge plus particulièrement le rôle des relations interindividuelles et des solidarités collectives dans le quotidien périurbain. Partant du constat « de la focalisation du regard des chercheurs sur la forme individualisée des déplacements périurbains », principale source des représentations négatives et parfois caricaturales dont le périurbain est l'objet, les auteurs soulignent pour leur part l'émergence «d'arrangements collectifs », non dépourvus de mécanismes de solidarité et potentiellement porteurs d'effets de territorialisation. En outre, les auteurs proposent de qualifier d' ' identité de mouvement » ce sentiment de reconnaissance et d'appartenance fondé sur le partage des mêmes conditions matérielles et idéelles de déplacement, qui se trouve selon eux au cœur de la compréhension de l'organisation individuelle et collective des mobilités. Le deuxième texte, de Benoît Feildel et al., s'intéresse pour sa part à l'imaginaire des mobilités et ses potentiels ressorts pour une mise en durabilité des espaces périurbains. Tandis que l'analyse des imaginaires spatiaux, en particulier dans les espaces périurbains, s'est principalement attachée jusqu'à présent aux espaces de résidence, les auteurs proposent quant à eux d'éclairer un point aveugle, celui des imaginaires attachés à la mobilité dans les périphéries urbaines - lorsque notamment leurs habitants se trouvent confrontés aux injonctions sociétales à la durabilité. S'attachant à l'analyse des discours habitants - récoltés sur la base d'un protocole d'enquête couplant des relevés GPS et des récits de mobilités - ils montrent la place qu'occupe l'imaginaire dans la construction du sens des mobilités et soulignent quelques perspectives pratiques pour un «(ré)enchantement social des mobilités » dans les espaces périurbains.

La deuxième partie est, elle aussi, constituée de deux contributions, dont la dimension principale mais pas unique est d'ordre méthodologique, visant ainsi à éclairer le sens objectivé des mobilités. Le premier texte, proposé par Denis Martouzet, repose sur l'articulation entre déplacements et mobilité tels que ces deux notions ont été préalablement définies l'une par rapport à l'autre. Cette articulation a aussi une dimension méthodologique pour capter les pratiques effectuées par les individus qui renvoient aux déplacements comme à la mobilité. C'est donc aussi celle d'une mesure quantitative objective fiable avec une approche qualitative, nécessairement plus incertaine quant à ses attendus, du rapport de l'individu à ses espaces et à ses mouvements. La mesure contient et contraint de ce fait le discours. Il met ainsi en évidence la nécessité de prendre en compte les mécanismes psychologiques, cognitifs pour saisir le sens des mobilités mais surtout comment la mesure objective réduit les biais liés à ces mécanismes qui échappent toujours en partie à leurs propres auteurs. Le second texte, de Matthieu Adam, renverse le propos. La mobilité n'est plus l'objectif à saisir, elle devient le moyen de saisie du sens de l'espace et de son devenir. Mettre en mouvement les habitants de deux quartiers en devenir, deux écoquartiers, à l'intérieur ou à proximité de ceux-ci, permet de reconsidérer la compréhension et les représentations que ces individus se font du quartier, les projections qu'ils y portent, par le fait même que le déplacement n'est jamais simple mouvement physique mais déjà, aussi, synthèse de valeurs qui s'expriment dans l'acte de se mouvoir.

Les deux textes suivants, constituant la troisième et dernière partie du dossier consacrée au sens pratique des mobilités, focalisent l'analyse sur des populations particulières : les jeunes placés en foyer en région urbaine, d'une part, les populations captives sur le plan de la mobilité dans les espaces ruraux délaissés, d'autre part. Leurs mobilités sont bien évidemment sans comparaison possible tant leurs caractéristiques sociales, individuelles, professionnelles, en termes de ressources financières, culturelles, cognitives, etc. sont différentes dans des contextes territoriaux eux-mêmes peu semblables. Pour autant, ce n'est pas cet ensemble de différences factuelles qui ressortent de la lecture de ces deux textes, c'est plutôt, à partir de l'analyse des mobilités qui en découlent, le regard que portent les pouvoirs publics ou leurs représentants sur les mobilités de ces catégories de personnes aux situations dissemblables. Ce regard institutionnel porté sur l'autre, simple individu, n'est pas simple analyse des déplacements ni même, plus largement, des mobilités. Elle est jugement de valeur. Le titre du texte de Fleur Guy est de ce point de vue très éclairant : «Ils bougent, ils bougent... mais jamais où il faut !». Finalement, par une imbrication finement menée, cet article nous propose d'avoir un regard qu'un lecteur peut poser sur le regard d'un chercheur lui-même observant le point de vue porté par une institution sur 
des personnes. Plus descriptive mais non moins efficace dans la déconstruction des jugements de valeur, l'étude de cas que propose Pierre Couturier s'attache à analyser le traitement institutionnel de la sous-mobilité rurale dans le Morvan nivernais et à mettre en lumière les réponses apportées à ce qui est pensé d'abord, voire uniquement, comme un problème par les autorités organisatrices des transports et plus largement les institutions en charge de l'aménagement et du fonctionnement des territoires. Il y a donc, implicitement, une évaluation sociale de situations diverses, toujours différentes et, explicitement, un traitement global qui est proposé, à l'échelle territoriale, fondé sur une certaine idée du fonctionnement de la société. Là aussi, par un jeu d'emboîtement, la compréhension de la mobilité des habitants par le chercheur permet de saisir le sens qu'en donnent les pouvoirs publics.

\section{Bibliographie}

1. Allemand $\mathrm{S}$ et al. (2004) Les sens du mouvement. Modernité et mobilité dans les sociétés urbaines contemporaines. Belin, Paris.

2. SIEF (2013) Circulation. $11^{\mathrm{e}}$ congrès international de la Société Internationale d'Ethnologie et de Folklore (SIEF). 30 juin - 4 juillet 2013. Tartu, Estonie.
3. Rastier F (2003) De la signification au sens. Pour une sémiotique sans ontologie. Texto ! [en ligne], http://www.revue-texto.net/ Inedits/Rastier_Semiotique-ontologie.hmtl

4. MSFS (2014) Métro, boulot, dodo... Quoi de neuf dans nos routines? 13 ${ }^{\mathrm{e}}$ colloque "Mobilité Spatiale, Fluidité Sociale 》 de l'Association internationale de sociologie de langue française (AISLF). 26 - 27 mars 2014. Lille, France.

5. Martouzet D (2013) Mobilités résidentielles, parcours professionnels et déplacements quotidiens: spatialités emboîtées et construction de l'habiter. In: Gerber $\mathrm{Ph}$, Carpentier $\mathrm{S}$ (éds) Mobilités et modes de vie: vers une recomposition de l'habiter. Presses Universitaires de Rennes, pp 49-67.

6. Depeau S, Ramadier T (2011) Se déplacer pour se situer. Places en jeu, enjeux de classes. Presses Universitaires de Rennes.

7. Hirschhorn M, Berthelot J-M (1996) Mobilités et ancrages. Vers un nouveau mode de spatialisation? L'Harmattan.

8. Kaufmann V (2008) Les paradoxes de la mobilité. Bouger, $s$ 'enraciner. Presses Polytechniques et Universitaires Romandes.

9. Carpentier S (2007) Une analyse exploratoire des liens entre mobilité quotidienne et ancrage résidentiel: vers une approche écologique de la mobilité? Articulo.ch - revue de sciences humaines, http://articulo.revues.org/619

10. Feildel B, Martouzet D (2012) La mobilité comme modalité de l'ancrage: enrichir l'évaluation de la durabilité des espaces périurbains. Recherche Transports Sécurité 28: 271-289.

11. Mathieu N (1996) Rural et urbain: unité et diversité dans les évolutions des modes d'habiter. In: Jollivet M, Eizner N (éds) L'Europe et ses campagnes. Presses de la Fondation nationale des sciences politiques, pp 187-216.

12. Morel-Brochet A, Ortar N (2012) La fabrique des modes d'habiter. Homme, lieux et milieux de vie. L'Harmattan. 